MATHEMATICS OF COMPUTATION

Volume 69, Number 231, Pages 1117-1130

S 0025-5718(00)01120-0

Article electronically published on February 17, 2000

\title{
EVALUATING HIGHER DERIVATIVE TENSORS BY FORWARD PROPAGATION OF UNIVARIATE TAYLOR SERIES
}

\author{
ANDREAS GRIEWANK, JEAN UTKE, AND ANDREA WALTHER
}

\begin{abstract}
This article considers the problem of evaluating all pure and mixed partial derivatives of some vector function defined by an evaluation procedure. The natural approach to evaluating derivative tensors might appear to be their recursive calculation in the usual forward mode of computational differentiation. However, with the approach presented in this article, much simpler data access patterns and similar or lower computational counts can be achieved through propagating a family of univariate Taylor series of a suitable degree. It is applicable for arbitrary orders of derivatives. Also it is possible to calculate derivatives only in some directions instead of the full derivative tensor. Explicit formulas for all tensor entries as well as estimates for the corresponding computational complexities are given.
\end{abstract}

\section{INTRODUCTION}

Many applications in scientific computing require second- and higher-order derivatives. Therefore, this article deals with the problem of calculating derivative tensors of some vector function

$$
\mathbf{y}=\mathbf{f}(\mathbf{x}) \quad \text { with } \quad \mathbf{f}: \mathcal{D} \subset \mathbb{R}^{\bar{n}} \mapsto \mathbb{R}^{m}
$$

that is the composition of (at least locally) smooth elementary functions. Assume that $\mathbf{f}$ is given by an evaluation procedure in $\mathrm{C}$ or some other programming language. Then $\mathbf{f}$ can be differentiated automatically [6]. The Jacobian matrix of first partial derivatives can be computed by the forward or reverse mode of the chainrule based technique known commonly as computational differentiation (CD). CD also yields second derivatives that are needed in optimization [8] and even higher derivatives that are called for in numerical bifurcation, beam dynamics [2] and other nonlinear calculations.

Even though the reverse mode of CD may be more efficient when the number of dependent variables is small compared to the number of independents [6], only the forward mode will be considered here. The mechanics of this direct application of the chain rule are completely independent of the number of dependent variables, so it is possible to restrict the analysis to a scalar-valued function

$$
y=f(\mathbf{x}) \quad \text { with } \quad f: \mathcal{D} \subset \mathbb{R}^{\bar{n}} \mapsto \mathbb{R} .
$$

Received by the editor January 2, 1998 and, in revised form, June 30, 1998.

1991 Mathematics Subject Classification. Primary 65D05, 65Y20, 68Q40.

Key words and phrases. Higher order derivatives, computational differentiation.

This work was partially supported by the Deutsche Forschungsgesellschaft under grant GR $705 / 4-1$

(C)2000 American Mathematical Society 
In other words, formulas for one component $f(\mathbf{x})$ of the original vector function $\mathbf{f}(\mathbf{x})$ are derived. This greatly simplifies the notation, and the full tensors can then easily be obtained by an outer loop over the component index.

The natural approach to evaluating derivative tensors seems to be their recursive calculation using the usual forward mode of CD. This technique has been implemented by Berz [3], Neidinger [10], and others. The only complication here is the need to utilize the symmetry in the higher derivative tensors, which leads to fairly complex addressing schemes. As has been mentioned in [7] and [1], much simpler data access patterns and similar or lower computational counts can be achieved through propagating a family of univariate Taylor series of an arbitrary degree. At the end, their values are interpolated to yield the tensor coefficients.

The paper is organized as follows. Section 2 introduces the notations that are used and makes some general observations. In Section 3 the complexity of storing and propagating multivariate and univariate Taylor polynomials is examined, and the advantages of the univariate Taylor series are shown. Section 4 derives formulas for the calculation of all mixed partial derivatives up to degree $d$ from a family of univariate Taylor polynomials of degree $d$. Some run-time results are presented in Section 5 Finally, Section 6 outlines some conclusions.

\section{Notations AND BASiC OBSERVATIONS}

In many applications, one does not require full derivative tensors but only the derivatives in $n \leq \bar{n}$ directions $\mathbf{s}_{i} \in \mathbb{R}^{\bar{n}}$. Therefore suppose a collection of $n \leq \bar{n}$ directions $\mathbf{s}_{i} \in \mathbb{R}^{\bar{n}}$ is given, and that they form a matrix

$$
\mathbf{S}=\left[\mathbf{s}_{1}, \mathbf{s}_{2}, \ldots, \mathbf{s}_{n}\right] \in \mathbb{R}^{\bar{n} \times n} .
$$

One possible choice is $\mathbf{S}=\mathbf{I}$ with $n=\bar{n}$. Here, $\mathbf{I}$ denotes the identity matrix in $\mathbb{R}^{n \times n}$. Of particular interest is the case $n=1$, where only derivatives in one direction are calculated.

With a coefficient vector $\mathbf{z} \in \mathbb{R}^{n}$, one may wish to calculate the derivative tensors

$$
\begin{aligned}
\left.\frac{\partial}{\partial \mathbf{z}} f(\mathbf{x}+\mathbf{S z})\right|_{\mathbf{z}=0} & =\left(f^{\prime}(\mathbf{x}) \mathbf{s}_{j}\right)_{j=1, \ldots, n} \in \mathbb{R}^{n}, \\
\left.\frac{\partial^{2}}{\partial \mathbf{z}^{2}} f(\mathbf{x}+\mathbf{S z})\right|_{\mathbf{z}=0} & =\left(f^{\prime \prime}(\mathbf{x}) \mathbf{s}_{i} \mathbf{s}_{j}\right)_{i=1, \ldots, n}^{j=1, \ldots, n} \in \mathbb{R}^{n \times n},
\end{aligned}
$$

and so on. The last equation is already an abuse of the usual matrix-vector notation. Here, the abbreviation

$$
\nabla_{S}^{k} f(\mathbf{x}) \in \mathbb{R}^{n^{k}}
$$

will be used in order to denote the $k$-th derivative tensor of $f(\mathbf{x}+\mathbf{S z})$ with respect to $\mathbf{z}$ at $\mathbf{z}=0$.

The use of the seed matrix $\mathbf{S}$ allows us to restrict our considerations to a subspace spanned by the columns $\mathbf{s}_{i}$ along which the properties of $f$ might be particularly interesting. This situation arises for example in optimization and bifurcation theory, where the range of $\mathbf{S}$ might be the tangent space of the active constraints or the nullspace of a degenerate Jacobian. Especially, when $n \ll \bar{n}$ it is obviously preferable to calculate the restricted tensors $\nabla_{\mathbf{S}}^{k} f(\mathbf{x})$ directly, rather than first evaluating the full tensors

$$
\nabla^{k} f(\mathbf{x}) \equiv \nabla_{I}^{k} f(\mathbf{x}) \in \mathbb{R}^{\bar{n}^{k}}
$$


and then contracting them by multiplications with the directions $\mathbf{s}_{i}$.

One way to evaluate the desired tensors $\nabla_{\boldsymbol{S}}^{k} f(\mathbf{x})$ is to recursively calculate for all intermediate quantities $w$ the corresponding restricted tensors $\nabla_{\boldsymbol{S}} w, \nabla_{\boldsymbol{S}}^{2} w, \ldots$ etc. The process would be started with the initialization $\nabla_{\boldsymbol{S}} \mathbf{x}=\mathbf{S}$ and with $\nabla_{\boldsymbol{S}}^{k} \mathbf{x}=0$ for $k>1$. For all subsequent intermediates, the derivative tensors are defined by the usual differentiation rules. For example, in the case $w=u * v$ there are the well known differentiation rules

$$
\nabla_{\boldsymbol{S}} w=u \nabla_{\boldsymbol{S}} v+v \nabla_{\boldsymbol{S}} u
$$

and

$$
\nabla_{\boldsymbol{S}}^{2} w=u \nabla_{\boldsymbol{S}}^{2} v+\nabla_{\boldsymbol{S}} u\left(\nabla_{\boldsymbol{S}} v\right)^{T}+\nabla_{\boldsymbol{S}} v\left(\nabla_{\boldsymbol{S}} u\right)^{T}+v \nabla_{\boldsymbol{S}}^{2} u
$$

For the third derivative tensor, the matrix-vector notation is no longer sufficient, but the following observation is generally applicable.

To calculate $\nabla_{\boldsymbol{S}}^{k} w$, each element of $\nabla_{\boldsymbol{S}}^{j} u$ with $0 \leq j \leq k$ has to be considered and then multiplied with all elements of $\nabla_{S}^{k-j} v$. The result is then multiplied by a binomial coefficient given by Leibniz' theorem, and finally incremented to the appropriate element of $\nabla_{\boldsymbol{S}}^{k} w$.

The next section studies the complexity of storing and propagating multivariate and univariate Taylor polynomials. Taylor coefficients are used since they are somewhat better scaled than the corresponding derivatives and satisfy slightly simpler recurrences.

\section{The COMPlexity of PROPAGATING UNIVARIATE TAYlor SERIES}

If the $k$-th tensor were stored and manipulated as a full $n^{k}$ array, the corresponding loops could be quite simple to code, but the memory requirement and operations count would be unnecessary high. In the case $k=2$, ignoring the symmetry of Hessians would almost double the storage per intermediate (from $\frac{1}{2}(n+1) n$ to $n^{2}$ ) and increase the operations count for a multiplication by about fifty percent. This price may be worth paying in return for the resulting sequential or at least constant stride data access. However, by standard combinatorial arguments

$$
\nabla_{\boldsymbol{S}}^{k} f(\mathbf{x}) \in \mathbb{R}^{n^{k}}
$$

has exactly

$$
\left(\begin{array}{c}
n+k-1 \\
k
\end{array}\right)=\frac{n \cdot(n+1) \cdots(n+k-1)}{1 \cdot 2 \cdots k} \approx \frac{n^{k}}{k !}
$$

distinct elements. Hence, the symmetry reduces the number of distinct elements in $\nabla_{\boldsymbol{S}}^{k} w$ almost by the factor $k$ !. Therefore in the case $k=3$ the number of distinct elements is reduced almost by six and in the case $k=4$ the storage ratio becomes almost 24. Since higher order tensors have very many entries, one has to utilize symmetric storage modes.

The drawback of symmetric storage modes is that the access of individual elements is somewhat complicated, requiring for example three integer operations for address computations in the implementation of Berz [3. Moreover, the resulting memory locations may be far apart with irregular spacing, so that significant paging overhead may be incurred. None of these difficulties arises when $n=1$. Then for any intermediate value $w$ the directional derivatives $w, \nabla_{\boldsymbol{s}} w, \nabla_{\boldsymbol{s}}^{2} w, \ldots, \nabla_{\boldsymbol{s}}^{d} w$ can be stored and manipulated as a contiguous array of $(d+1)$ scalars. Here $d$ denotes 
the highest degree that is desired. It will be shown in Section 4 that to reconstruct the full tensors $\nabla_{\boldsymbol{S}}^{k} f(\mathbf{x})$ for $k=0, \ldots, d$, exactly as many univariate Taylor series are needed as the highest tensor $\nabla_{S}^{d} f(\mathbf{x})$ has distinct elements. As we will see, that entails a slight increase in storage, but a very significant gain in code simplicity and efficiency. After this outlook, the manipulation of Taylor polynomials is considered. The collection of Taylor coefficients that constitute the tensors

$$
\nabla_{\boldsymbol{S}}^{k} f(\mathbf{x}), \quad k=0, \ldots, d,
$$

represents a general polynomial of degree $d$ in $n$ variables. As can be concluded from (11), $\nabla_{S}^{k} f(\mathbf{x})$ contains

$$
\left(\begin{array}{c}
n+d \\
d
\end{array}\right)=\sum_{k=0}^{d}\left(\begin{array}{c}
n+k-1 \\
k
\end{array}\right)
$$

distinct monomials. The truncated Taylor polynomials of all intermediate scalar quantities $w$ have exactly the same structure. Considering again a product $w=u * v$, for the computation of $\nabla_{S}^{k} w$ each element of $\nabla_{S}^{j} u, 0 \leq j \leq k$, has to be multiplied with all elements of $\nabla_{S}^{k-j} v$. It follows that

$$
\left(\begin{array}{c}
2 n+k-1 \\
k
\end{array}\right)=\sum_{j=0}^{k}\left(\begin{array}{c}
n+j-1 \\
j
\end{array}\right)\left(\begin{array}{c}
n+k-j-1 \\
k-j
\end{array}\right)
$$

multiplications are necessary because of (1). Hence, the total count of multiplications for computing $\nabla_{S}^{d} w$ is

$$
\left(\begin{array}{c}
2 n+d \\
d
\end{array}\right)=\sum_{k=0}^{d}\left(\begin{array}{c}
2 n+k-1 \\
k
\end{array}\right) .
$$

When $n>1$ there are also a significant number of additions and other overhead for each multiplication. Nevertheless it is possible to consider the number

$$
\left(\begin{array}{c}
2 n+d \\
d
\end{array}\right) \approx \frac{1}{d !}(2 n)^{d} \quad \text { if } \quad d \ll n
$$

as a reasonable approximation for the factor by which the cost to evaluate $f$ grows when the calculation is performed in Taylor arithmetic of degree $d$ and order $n$. Here, we have tacitly used the fact that propagating Taylor polynomials through nonlinear functions such as the exponential, logarithm, and trigonometric functions is about as costly as the convolution for the product discussed above. All linear operations are cheaper, of course, since for them the effort is roughly $\left(\begin{array}{c}n+d \\ d\end{array}\right)$ and thus about the same as the number of data entries that need to be fetched and stored from and into memory.

Provided there is a significant fraction of nonlinear elementary functions in the overall calculation, one may consider

$$
\left(\begin{array}{c}
2 n+d \\
d
\end{array}\right) /\left(\begin{array}{c}
n+d \\
d
\end{array}\right) \approx 2^{d}
$$

as an approximate computation/communication ratio for propagating Taylor polynomials. Consequently, even on a modern super-scalar processor with comparatively slow memory access, communication should not be the bottleneck.

Now suppose that instead of propagating one Taylor polynomial in $n$ variables and degree $d$ one propagates $\left(\begin{array}{c}n+d-1 \\ d\end{array}\right)$ univariate Taylor polynomials of the same 
degree $d$. Since the common constant term needs to be stored only once, the amount of data per intermediate becomes

$$
1+\left(\begin{array}{c}
n+d-1 \\
d
\end{array}\right) d=1+\frac{d n}{d+n}\left(\begin{array}{c}
n+d \\
d
\end{array}\right) .
$$

By inspection of the right-hand side we see this is at most $d$ times that for the standard case (see (2)). Furthermore,

$$
\frac{(d+2)(d+1)}{2}=\left(\begin{array}{c}
d+2 \\
d
\end{array}\right)
$$

multiplications are needed to propagate one univariate Taylor polynomial of degree $d$ through an elementary multiplication. Hence, the total amount of the computational effort for propagating $\left(\begin{array}{c}n+d-1 \\ d\end{array}\right)$ univariate Taylor polynomials of degree $d$ is essentially given by

$$
\left(\begin{array}{c}
n+d-1 \\
d
\end{array}\right)\left(\begin{array}{c}
d+2 \\
d
\end{array}\right)=q(d, n)\left(\begin{array}{c}
2 n+d \\
d
\end{array}\right)
$$

where

$$
q(d, n) \equiv \frac{(d+2)(d+1)}{2} \cdot \frac{(n+d-1) \ldots(n)}{(2 n+d)(2 n+d-1) \ldots(2 n+1)} .
$$

It is easy to check through induction that $q$ is never greater than $\frac{3}{2}$ and that

$$
q(d) \equiv \lim _{n \rightarrow \infty} q(d, n)=\frac{(d+2)(d+1)}{2^{d+1}} .
$$

One has in particular $q(0, n)=1$ and

$$
\begin{aligned}
& q(1, n)=\frac{3}{2}-\frac{3}{4 n+2}=\frac{3}{2}-\mathcal{O}\left(\frac{1}{n}\right), \\
& q(2, n)=\frac{3}{2}-\frac{3}{4 n+2}=\frac{3}{2}-\mathcal{O}\left(\frac{1}{n}\right), \\
& q(3, n)=\frac{5 n(n+2)}{(2 n+3)(2 n+1)}=\frac{5}{4}-\mathcal{O}\left(\frac{1}{n}\right), \\
& q(4, n)=\frac{15 n(n+3)}{4(2 n+3)(2 n+1)} \quad=\quad \frac{15}{16}-\mathcal{O}\left(\frac{1}{n}\right), \\
& q(5, n)=\frac{21 n(n+3)(n+4)}{4(2 n+5)(2 n+3)(2 n+1)}=\frac{21}{32}-\mathcal{O}\left(\frac{1}{n}\right),
\end{aligned}
$$

as well as, for all higher derivatives $d \geq 6$,

$$
q(d, n)=\frac{(d+2)(d+1)}{2^{d+1}}+\mathcal{O}\left(\frac{1}{n}\right) .
$$

In other words, the computational effort is dramatically reduced when the degree $d$ is quite large. This fact can also be seen in Figure 1 which displays the function $q(d, n)$. For the probably more important moderate values of $d \leq 5$ the complexity ratio is surprisingly close to one. The computations/communications ratio is about $d / 2$, almost independently of $n$ (see (3) and (4)). For $d \leq 4$ that ratio might appear rather small. However, the memory access is now strictly sequential, and no addressing calculations are needed.

In this section, we have demonstrated that for moderate values of $d$ the propagation of $\left(\begin{array}{c}n+d-1 \\ d\end{array}\right)$ univariate Taylor series has essentially the same operations count as multivariate derivative propagation. We found also that for higher degree derivatives, one needs considerable fewer operations using univariate Taylor series instead of the multivariate one. In the next section, an efficient scheme for interpolating 


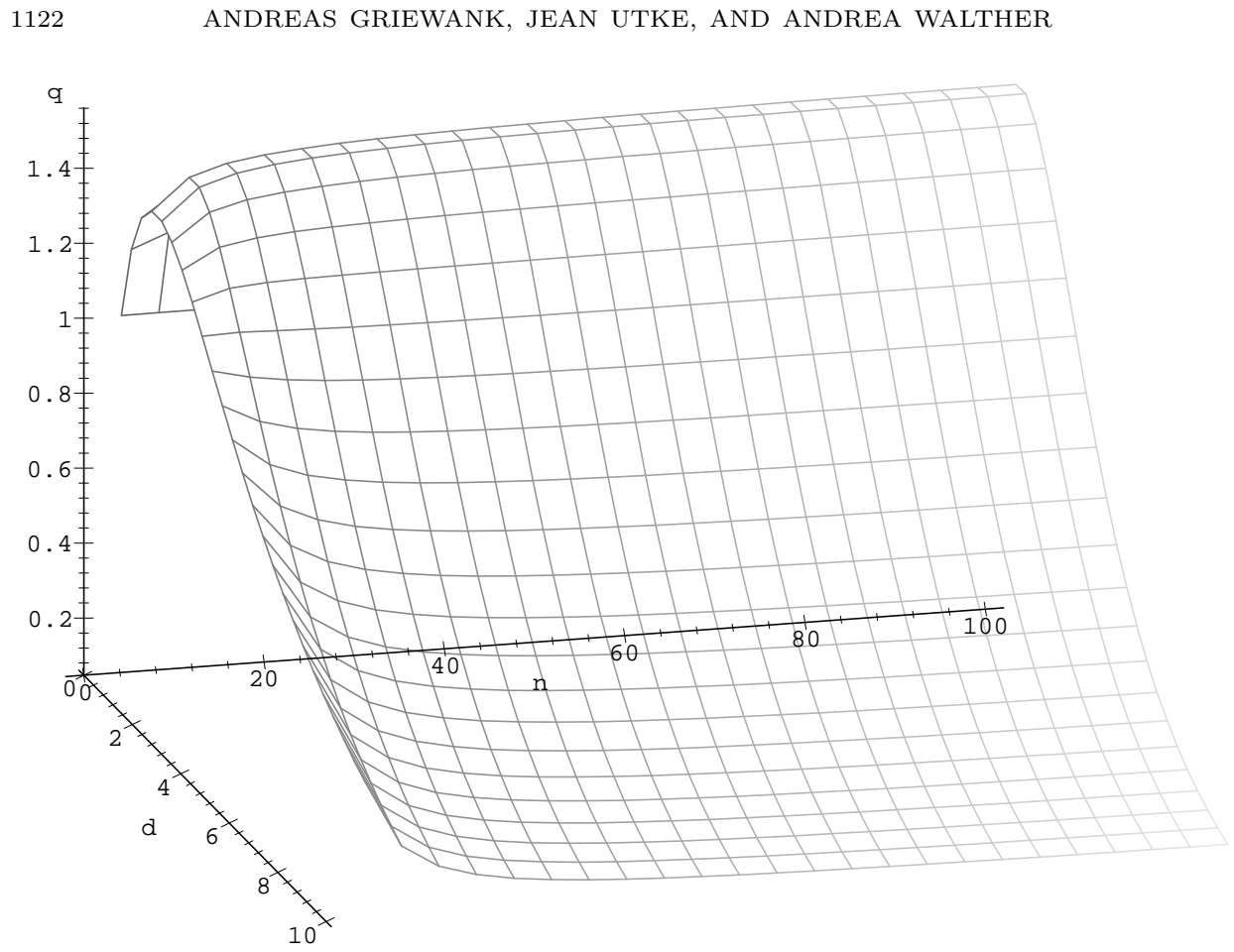

FigURE 1. $q(d, n)$ for $n=4, \ldots, 100, d=0, \ldots, 10$

all partial derivatives up to degree $d$ from these $\left(\begin{array}{c}n+d-1 \\ d\end{array}\right)$ univariate Taylor series is developed.

\section{Interpolation With Univariate TAYlor SERIES}

For each direction $\mathbf{s} \in \mathbb{R}^{\bar{n}}$ one can obtain the Taylor expansion

(5) $f(\mathbf{x}+t \mathbf{s})=f(\mathbf{x})+f^{(1)}(\mathbf{x} ; \mathbf{s}) t+f^{(2)}(\mathbf{x} ; \mathbf{s}) t^{2}+\cdots+f^{(d)}(\mathbf{x} ; \mathbf{s}) t^{d}+\mathcal{O}\left(t^{d+1}\right)$.

The notation $f^{(m)}(\mathbf{x} ; \mathbf{s})$ will be used throughout to denote the $m$-th homogeneous polynomial in $\mathbf{s}$ in the Taylor expansion of $f$ at $\mathbf{x}$. Hence, $\mathbf{x}$ is considered constant and $\mathbf{s} \in \mathbb{R}^{\bar{n}}$ variable with the homogeneity property

$$
f^{(m)}(\mathbf{x} ; t \mathbf{s})=t^{m} f^{(m)}(\mathbf{x} ; \mathbf{s}) \quad \text { for } t \in \mathbb{R} .
$$

Since only the last coefficient $f^{(d)}(\mathbf{x} ; \mathbf{s})$ contains any information about the highest tensor $\nabla_{\boldsymbol{s}}^{d} f(\mathbf{x})$ with its $\left(\begin{array}{c}n+d-1 \\ d\end{array}\right)$ distinct elements, it is clear that one needs to evaluate at least that many univariate expansions. We will see that this number is sufficient.

Let $\mathbf{i}=\left(\mathbf{i}_{1}, \ldots, \mathbf{i}_{n}\right) \in \mathbb{N}_{0}^{n}$ with $\mathbf{i}_{j} \in \mathbb{N}_{0}(j=1, \ldots, n)$ be a multi-index. The norm of $\mathbf{i}$ is defined by

$$
|\mathbf{i}|=\sum_{j=1}^{n} \mathbf{i}_{j} .
$$

Consider the matrix $\mathbf{S}$ throughout this section as fixed. Now choose the directions

$$
\text { Si } \quad \forall \mathbf{i} \in \mathbb{N}_{0}^{n} \text { with }|\mathbf{i}|=d .
$$


After selecting a particular $d$, Taylor coefficients are propagated along these $\left(\begin{array}{c}n+d-1 \\ d\end{array}\right)$ necessary directions only. In other words, for each multi-index $\mathbf{i} \in \mathbb{N}_{0}^{n}$ with $|\mathbf{i}|=d$, we consider the Taylor series for the univariate function

$$
\varphi_{\mathbf{i}}: \mathbb{R} \mapsto \mathbb{R}, \quad \varphi_{\mathbf{i}}(t) \equiv f(\mathbf{x}+t \mathbf{S i}),
$$

and evaluate the Taylor coefficients of $\varphi_{\mathbf{i}}$ up to the degree $d$. The dependence of the $\varphi_{\mathbf{i}}$ on $\mathbf{S}$ is not denoted explicitly, because $\mathbf{S}$ is considered as fixed.

Note that there is no propagation along the directions $\mathbf{S i}$ with $|\mathbf{i}|<d$. However, we will see that it is still possible to obtain all lower order derivative information that is necessary to compute the tensors $\nabla_{\boldsymbol{S}}^{m} f$ with $m<d$.

Interpolating second derivatives. To illustrate our approach, let us first consider the computation of the Hessian $\nabla_{\boldsymbol{S}}^{2} f$ with $\mathbf{S}=\mathbf{I}$ when the maximal degree $d$ equals 2. Denote the $i$-th unit vector in $\mathbb{R}^{n}$ by $\mathbf{e}_{i}$. Then, the restricted gradient components of $\nabla_{\boldsymbol{S}} f(\mathbf{x})$ are obtained immediately as

$$
\nabla_{\boldsymbol{S}} f(\mathbf{x}) \mathbf{e}_{i}=\frac{1}{2} \varphi_{\mathbf{i}}^{\prime}(0)=\frac{1}{2} \nabla f(\mathbf{x}) \mathbf{S i} \quad \text { with } \quad \mathbf{i} \in \mathbb{N}_{0}^{n}, \mathbf{i}_{k}= \begin{cases}2, & k=i \\ 0, & k \neq i\end{cases}
$$

Similarly, the pure second derivatives can be obtained from

$$
\mathbf{e}_{i}^{T} \nabla_{\mathbf{S}}^{2} f(\mathbf{x}) \mathbf{e}_{i}=\frac{1}{4} \varphi_{\mathbf{i}}^{\prime \prime}(0)=\frac{1}{4}(\mathbf{S i})^{T} \nabla^{2} f(\mathbf{x}) \mathbf{S i}
$$

with the same multi-index $\mathbf{i}$ as in (7). However, the mixed second derivatives $\mathbf{e}_{i}^{T} \nabla_{\mathbf{S}}^{2} f(\mathbf{x}) \mathbf{e}_{j}, i \neq j$, are not directly available. To get them, one has to consider the diagonal direction $\mathbf{S l}$ defined by the multi-index

$$
\mathbf{l} \in \mathbb{N}_{0}^{n} \text { with } \mathbf{l}_{k}=\left\{\begin{array}{l}
1 \text { if } k=i \text { or } k=j \\
0 \text { if } k \neq i \text { and } k \neq j .
\end{array}\right.
$$

Because of $|\mathbf{1}|=2$ the second order Taylor coefficient of $\varphi_{\mathbf{l}}$ is known:

$$
\begin{aligned}
\varphi_{\mathbf{l}}^{\prime \prime}(0) & =\left(\mathbf{e}_{i}+\mathbf{e}_{j}\right)^{T} \nabla_{\mathbf{S}}^{2} f(\mathbf{x})\left(\mathbf{e}_{i}+\mathbf{e}_{j}\right) \\
& =\mathbf{e}_{i}^{T} \nabla_{\mathbf{S}}^{2} f(\mathbf{x}) \mathbf{e}_{i}+\mathbf{e}_{j}^{T} \nabla_{\mathbf{S}}^{2} f(\mathbf{x}) \mathbf{e}_{j}+2 \mathbf{e}_{i}^{T} \nabla_{\boldsymbol{S}}^{2} f(\mathbf{x}) \mathbf{e}_{j} .
\end{aligned}
$$

This identity yields the interpolation formula

$$
\begin{aligned}
\mathbf{e}_{i}^{T} \nabla_{\boldsymbol{S}}^{2} f(\mathbf{x}) \mathbf{e}_{j} & =\frac{1}{2}\left[\left(\mathbf{e}_{i}+\mathbf{e}_{j}\right)^{T} \nabla_{\boldsymbol{S}}^{2} f(\mathbf{x})\left(\mathbf{e}_{i}+\mathbf{e}_{j}\right)-\mathbf{e}_{i}^{T} \nabla_{\boldsymbol{S}}^{2} f(\mathbf{x}) \mathbf{e}_{i}-\mathbf{e}_{j}^{T} \nabla_{\boldsymbol{S}}^{2} f(\mathbf{x}) \mathbf{e}_{j}\right] \\
& =\frac{1}{2} \varphi_{\mathbf{l}}^{\prime \prime}(0)-\frac{1}{8} \varphi_{\mathbf{i}}^{\prime \prime}(0)-\frac{1}{8} \varphi_{\mathbf{j}}^{\prime \prime}(0)
\end{aligned}
$$

with the multi-indices $\mathbf{i}, \mathbf{j}, \mathbf{l} \in \mathbb{N}_{0}^{n}$ and

$$
\mathbf{i}_{k}=\left\{\begin{array}{l}
2 \text { if } k=i, \\
0 \text { if } k \neq i,
\end{array} \quad \mathbf{j}_{k}=\left\{\begin{array}{l}
2 \text { if } k=j, \\
0 \text { if } k \neq j,
\end{array} \quad \mathbf{l}_{k}=\left\{\begin{array}{l}
1 \text { if } k=i \text { or } k=j, \\
0 \text { if } k \neq i \text { and } k \neq j .
\end{array}\right.\right.\right.
$$

When $d=3$ this scheme is not directly applicable because $|\mathbf{1}|<3$. Therefore $\mathbf{l}$ does not aim at a derivative of the highest order, and one does not propagate a Taylor series along the direction Sl. Hence, $\varphi_{1}^{\prime \prime}(0)$ is unknown. To handle that situation and generally the higher order cases, one needs a systematic way of generating formula of the form (8) for mixed partials. 
Computing mixed partials from grid values. For any polynomial $P$ of degree $n$ or less, it can be checked that

$$
\frac{\partial^{n} P(\mathbf{S z})}{\partial z_{1} \partial z_{2} \ldots \partial z_{n}}=\sum_{\mathbf{i}_{1}=0}^{1} \cdots \sum_{\mathbf{i}_{n}=0}^{1} P\left(\mathbf{i}_{1} \mathbf{s}_{1}+\cdots+\mathbf{i}_{n} \mathbf{s}_{n}\right)(-1)^{n-\left(\mathbf{i}_{1}+\cdots+\mathbf{i}_{n}\right)}
$$

through integration of the constant function on the left side over the unit cube in $n$ dimensions. The important observation here is that one only has to know the value of the polynomial at the corners of the parallelepiped $\left\{\mathbf{S z}: 0 \leq \mathbf{z}_{i} \leq 1\right\}$ in order to compute the mixed derivative with respect to all $\mathbf{z}_{i}$. Naturally, one does not want to assume that $f$ itself is a polynomial. However, one can use the $m$-th coefficients of the univariate Taylor expansions to compute values of the homogeneous approximating polynomials $f^{(m)}(\mathbf{x} ; \mathbf{s}), 1 \leq m \leq d$, satisfying (5) and (6). For example, it follows immediately for the second mixed partials with $P(\mathbf{S z})=f^{(2)}(\mathbf{x} ; \mathbf{S z})$ and $1 \leq j<i \leq n$ :

$$
\frac{\partial^{2} f^{(2)}(\mathbf{x} ; \mathbf{S z})}{\partial z_{i} \partial z_{j}}=\underbrace{f^{(2)}\left(\mathbf{x} ; \mathbf{S}\left(\mathbf{e}_{i}+\mathbf{e}_{j}\right)\right)}_{=\frac{1}{2} \varphi_{1}^{\prime \prime}(0)}-\underbrace{f^{(2)}\left(\mathbf{x} ; \mathbf{S e}_{i}\right)}_{=\frac{1}{8} \varphi_{\mathbf{i}}^{\prime \prime}(0)}-\underbrace{f^{(2)}\left(\mathbf{x} ; \mathbf{S e}_{j}\right)}_{=\frac{1}{8} \varphi_{\mathbf{j}}^{\prime \prime}(0)}+f^{(2)}(\mathbf{x} ; 0)
$$

with the multi-indices $\mathbf{i}, \mathbf{j}$, and $\mathbf{l}$ defined as in (91). Through the homogeneity property (16) one obtains that

$$
f^{(m)}(\mathbf{x} ; 0)=0, \quad \forall m>0,
$$

so that the last term in (11) drops out and one gets

$$
\left.\frac{\partial^{2} f^{(2)}(\mathbf{x} ; \mathbf{S z})}{\partial z_{i} \partial z_{j}} \equiv \frac{\partial^{2} f(\mathbf{x}+\mathbf{S} \mathbf{z})}{\partial z_{i} \partial z_{j}}\right|_{\mathbf{z}=0}
$$

because of the previous formula (8). For fixed $\mathbf{S}$ and given $d$, the real numbers $f^{(|\mathbf{i}|)}(\mathbf{x} ; \mathbf{S i})$ with $|\mathbf{i}| \leq d$ will be referred to as the grid values.

By considering seed matrices $\mathbf{S} \in \mathbb{R}^{\bar{n} \times d}$ with repeated columns, one can derive from (10), for any polynomial $P$ of degree up to $d$, its generalization

$$
\frac{\partial^{|\mathbf{i}|} P(\mathbf{S} \mathbf{z})}{\partial z_{1}^{\mathbf{i}_{1}} \partial z_{2}^{\mathbf{i}_{2}} \ldots \partial z_{n}^{\mathbf{i}_{n}}}=\sum_{\mathbf{k}_{1}=0}^{\mathbf{i}_{1}} \sum_{\mathbf{k}_{2}=0}^{\mathbf{i}_{2}} \ldots \sum_{\mathbf{k}_{n}=0}^{\mathbf{i}_{n}}\left(\begin{array}{l}
\mathbf{i}_{1} \\
\mathbf{k}_{1}
\end{array}\right)\left(\begin{array}{c}
\mathbf{i}_{2} \\
\mathbf{k}_{2}
\end{array}\right) \cdots\left(\begin{array}{c}
\mathbf{i}_{n} \\
\mathbf{k}_{n}
\end{array}\right)(-1)^{|\mathbf{i}-\mathbf{k}|} P(\mathbf{S k}),
$$

where now $\mathbf{S} \in \mathbb{R}^{\bar{n} \times n}$ and $\mathbf{i}$ may be any multi-index with $|\mathbf{i}|=\operatorname{deg}(P)$. Applying this identity to the homogeneous components $f^{(|\mathbf{i}|)}(\mathbf{x} ; \mathbf{s})$ of $f$ at $\mathbf{x}$ and using binomial coefficient notation for multi-indices, one obtains, with (12) and the zero vector $\mathbf{0} \in \mathbb{N}_{0}^{n}$,

$$
\left.f_{\mathbf{i}}(\mathbf{x}) \equiv \frac{\partial^{|\mathbf{i}|} f(\mathbf{x}+\mathbf{S} \mathbf{z})}{\partial z_{1}^{\mathbf{i}_{1}} \partial z_{2}^{\mathbf{i}_{2}} \ldots \partial z_{n}^{\mathbf{i}_{n}}}\right|_{\mathbf{z}=0}=\sum_{\mathbf{0}<\mathbf{k} \leq \mathbf{i}}\left(\begin{array}{l}
\mathbf{i} \\
\mathbf{k}
\end{array}\right)(-1)^{|\mathbf{i}-\mathbf{k}|} f^{(|\mathbf{i}|)}(\mathbf{x} ; \mathbf{S k}) .
$$

We conclude this subsection with a geometric illustration of the situation. Suppose one wishes to obtain the partials $f_{\mathbf{i}}$ for all $\mathbf{i}$ with $1 \leq|\mathbf{i}| \leq d$. The functions $f^{(m)}(\mathbf{x} ; \mathbf{s}), \mathbf{s} \in \mathbb{R}^{\bar{n}}$, denote the $m$-th homogeneous polynomial at $\mathbf{x}$ as in equation (5). Through formula (13) it is possible to calculate $f_{\mathbf{i}}(\mathbf{x})$ from the grid values $f^{(|\mathbf{i}|)}(\mathbf{x} ; \mathbf{S k})$ for all $\mathbf{k} \leq \mathbf{i}$. However, the propagation of univariate Taylor series only yields some of the grid values directly. The others must be calculated in a second interpolation step. The values $f^{(m)}(\mathbf{x} ; \mathbf{S i})$ are known for all $|\mathbf{i}|=d$ and 


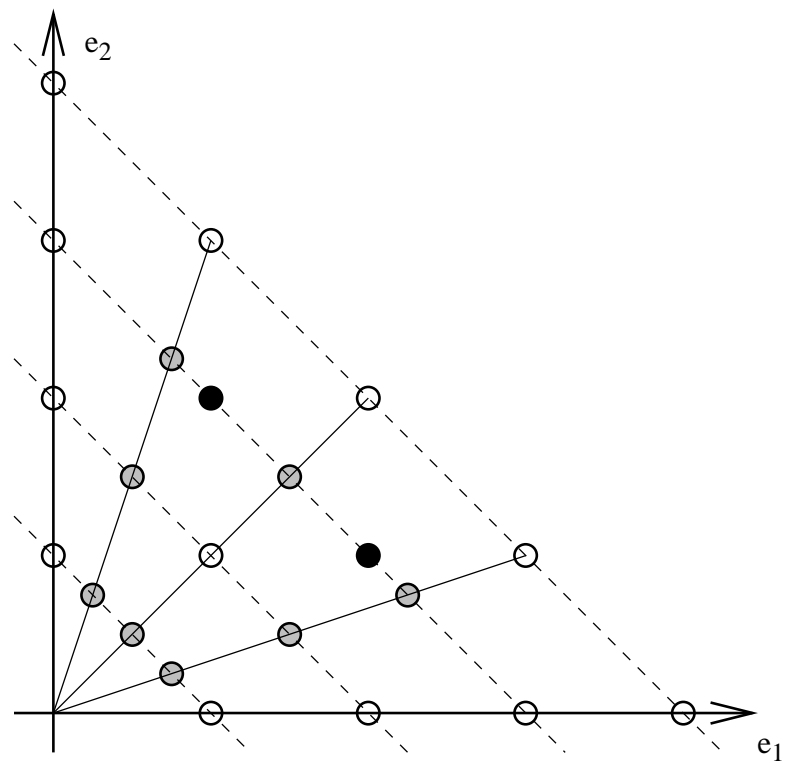

Figure 2. Grid values and ray values for $n=2$ and $d=4$

for all $0 \leq m \leq d$. They will be referred to as ray values. Figure 2 illustrates this situation for $n=2$ and $d=4$. The thin lines represent the directions of the propagated Taylor series including the $\mathbf{e}_{1^{-}}$and the $\mathbf{e}_{2}$-axes. The grid points whose values can be obtained by scaling of the ray values $f^{(m)}(\mathbf{x} ; \mathbf{S i})$ with $|\mathbf{i}|=d$ and $0 \leq m \leq d$ are marked by unfilled circles. The black circles denote the values that are desired because they arise on the right hand side of (13) but are as yet unknown. The grey shaded circles mark points that do not belong to the grid but whose values can be obtained by scaling.

The following section describes the way to compute the unknown grid values from the known ray values.

Computing grid values from ray values. For all multi-indices $\mathbf{k}$ with $|\mathbf{k}|<d$, the fact that $f^{(m)}(\mathbf{x} ; \mathbf{s})$ is homogeneous of degree $m$ implies

$$
f^{(m)}(\mathbf{x} ; \mathbf{S k})=(|\mathbf{k}| / d)^{m} f^{(m)}(\mathbf{x} ; \mathbf{S}(d \mathbf{k} /|\mathbf{k}|)) .
$$

The ray values are placed at equal distances, and the polynomial $\left(\begin{array}{c}\mathbf{z} \\ \mathbf{j}\end{array}\right) f^{(m)}(\mathbf{x} ; \mathbf{S} \mathbf{j})$ in $z$ is nonzero for only one ray point. Hence, an interpolation process similar to the one dimensional Lagrange interpolation formula yields the equation

$$
f^{(m)}(\mathbf{x} ; \mathbf{S z})=\sum_{|\mathbf{j}|=d}\left(\begin{array}{l}
\mathbf{z} \\
\mathbf{j}
\end{array}\right) f^{(m)}(\mathbf{x} ; \mathbf{S} \mathbf{j})
$$

for $\mathbf{z}=d \mathbf{k} /|\mathbf{k}|$ or any other vector $\mathbf{z} \in \mathbb{R}^{n}$ with value $|\mathbf{z}|=d$. Therefore the ray values $f^{(m)}(\mathbf{x} ; \mathbf{S}(d \mathbf{k} /|\mathbf{k}|))$ can be computed for any $\mathbf{k}$ with $|\mathbf{k}|<d$. Substituting 
(15) into (14), one obtains

$$
f^{(m)}(\mathbf{x} ; \mathbf{S k})=\sum_{|\mathbf{j}|=d}\left(\frac{|\mathbf{k}|}{d}\right)^{m}\left(\begin{array}{c}
d \mathbf{k} /|\mathbf{k}| \\
\mathbf{j}
\end{array}\right) f^{(m)}(\mathbf{x} ; \mathbf{S} \mathbf{j}) .
$$

Direct computation of mixed partials from ray values. Now the main result of this article can be established, namely an efficient scheme for calculating all mixed partial derivatives up to degree $d$ from the known ray values. The following proposition contains the explicit formula and an estimation of the complexity of the interpolation procedure.

Proposition 4.1. Let $f$ be at least d-times continuously differentiable at some point $\mathbf{x} \in \mathbb{R}^{\bar{n}}$. Then for any $\mathbf{i} \in \mathbb{N}_{0}^{n}$ with $1 \leq|\mathbf{i}| \leq d$ the partial derivative $f_{\mathbf{i}}(\mathbf{x})$ defined in (13) is given by the formula

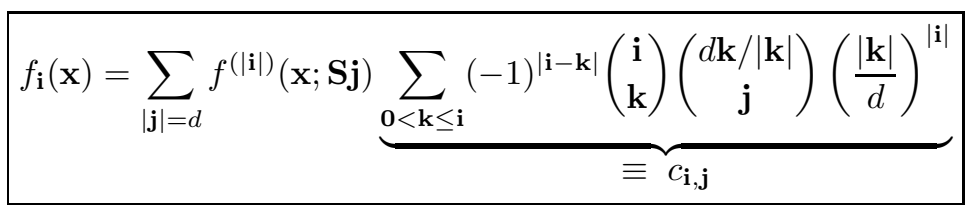

The number of nonvanishing coefficients

$$
0 \neq c_{\mathbf{i}, \mathbf{j}} \quad \text { for } \mathbf{i}, \mathbf{j} \in \mathbb{N}_{0}^{n}, 1 \leq|\mathbf{i}| \leq d,|\mathbf{j}|=d,
$$

is less than or equal to

$$
p(d, n) \equiv \sum_{m=1}^{d}\left(\begin{array}{l}
n \\
m
\end{array}\right)\left(\begin{array}{l}
d \\
m
\end{array}\right)\left(\begin{array}{c}
m+d-1 \\
d
\end{array}\right),
$$

which bounds the number of operations needed to calculate all partial derivatives from the univariate Taylor series.

Proof. Combining (13) and (16), one obtains the identity (17), which can be written in a simpler form as

$$
f_{\mathbf{i}}(\mathbf{x})=\sum_{|\mathbf{j}|=d} c_{\mathbf{i}, \mathbf{j}} f^{(|\mathbf{i}|)}(\mathbf{x} ; \mathbf{S} \mathbf{j})
$$

Define the sign function for multi-indices componentwise as follows:

$$
\operatorname{sign}(\mathbf{i}) \equiv\left(\operatorname{sign}\left(\mathbf{i}_{1}\right), \ldots, \operatorname{sign}\left(\mathbf{i}_{n}\right)\right) \quad \forall \mathbf{i} \in \mathbb{N}_{0}^{n} .
$$

For two multi-indices $\mathbf{i}$ and $\mathbf{j}$ with $1 \leq|\mathbf{i}| \leq d$ and $|\mathbf{j}|=d$, the coefficient $c_{\mathbf{i}, \mathbf{j}}$ can only be nonzero if

$$
\operatorname{sign}(\mathbf{j}) \leq \operatorname{sign}(\mathbf{i})
$$

since otherwise the second binomial coefficient in (17) must vanish for all $\mathbf{0}<\mathbf{k} \leq \mathbf{i}$. Now, consider an arbitrary $m \in \mathbb{N}$ with $1 \leq m \leq d$. A multi-index i with $|\operatorname{sign}(\mathbf{i})|=$ $m$ has $m$ nonvanishing components. Therefore the inequality $m \leq|\mathbf{i}| \leq d$ must hold for all $\mathbf{i}$ with $|\operatorname{sign}(\mathbf{i})|=m$. The number of distinct possibilities for choosing $m$ positive integers so that their sum is no greater than $d$ is given by $\left(\begin{array}{c}d \\ m\end{array}\right)$.

It follows that the total number of multi-indices $\mathbf{i}$ with $1 \leq|\mathbf{i}| \leq d$ and $|\operatorname{sign}(\mathbf{i})|=$ $m$ is equal to $\left(\begin{array}{c}n \\ m\end{array}\right)\left(\begin{array}{c}d \\ m\end{array}\right)$. For each of these multi-indices the coefficient $c_{\mathbf{i}, \mathbf{j}}$ can be greater than zero only if $\mathbf{j}$ satisfies the necessary condition (20). This implies that

$$
|\operatorname{sign}(\mathbf{j})| \leq m .
$$




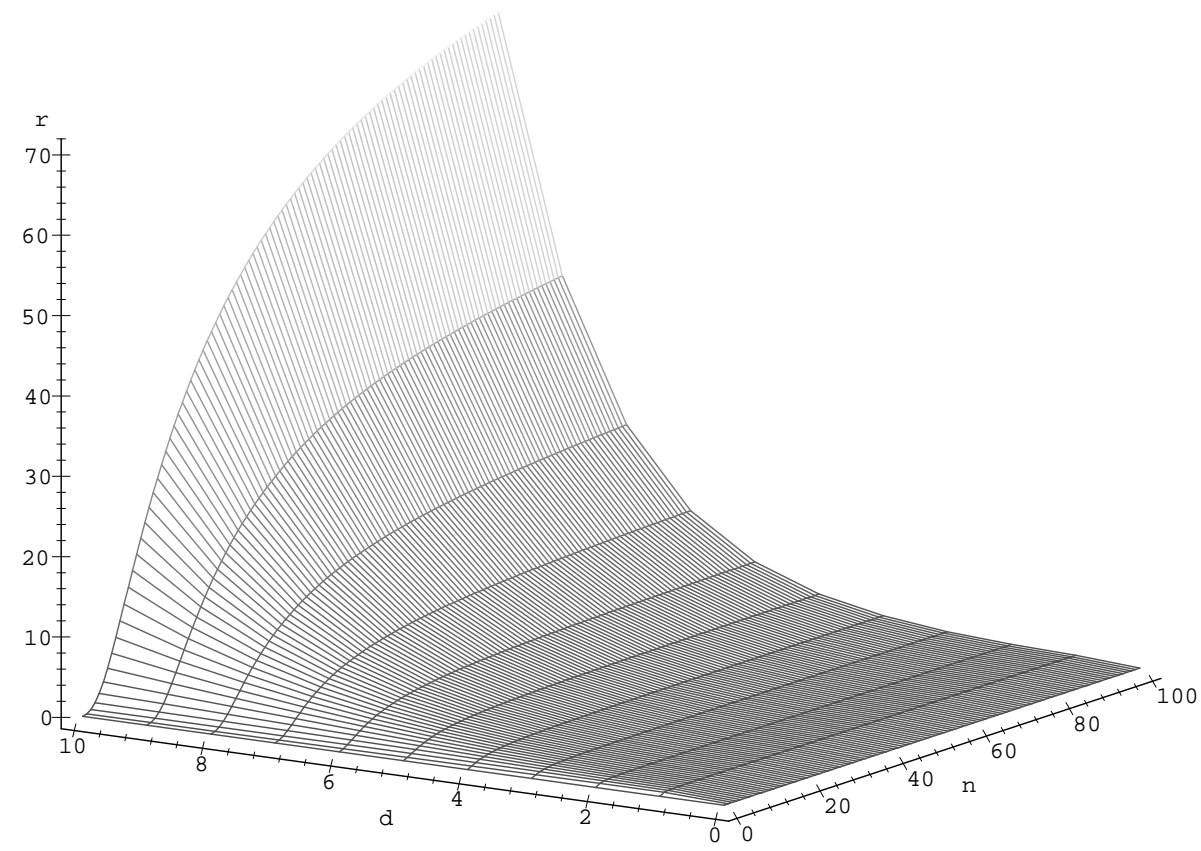

Figure 3. $r(d, n)$ for $n=1, \ldots, 100, d=0, \ldots, 10$

Therefore, one has that for each multi-index $\mathbf{i}$ with $1 \leq|\mathbf{i}| \leq d$ and $|\operatorname{sign}(\mathbf{i})|=m$ the number of multi-indices $\mathbf{j}$ with $\operatorname{sign}(\mathbf{j}) \leq \operatorname{sign}(\mathbf{i})$ is given by $\left(\begin{array}{c}m+d-1 \\ d\end{array}\right)$. Hence we conclude that the function

$$
p(d, n) \equiv \sum_{m=1}^{d}\left(\begin{array}{c}
n \\
m
\end{array}\right)\left(\begin{array}{c}
d \\
m
\end{array}\right)\left(\begin{array}{c}
m+d-1 \\
d
\end{array}\right)
$$

denotes an upper bound for the number of nonvanishing coefficients $c_{\mathbf{i}, \mathbf{j}}$. One can derive from (19) that (21) is also an upper bound for the total number of multiplications of the interpolation procedure.

It follows from the tables at the end of this section that for $d=2,3$ and any $n \in \mathbb{N}$, the function $p(d, n)$ gives the exact number of nonvanishing coefficients $c_{\mathbf{i}, \mathbf{j}}$. Therefore it may be conjectured that $p(d, n)$ corresponds exactly to the number of nonvanishing factors $c_{\mathbf{i}, \mathbf{j}}$, but so far there is no proof of this. Define

$$
r(d, n) \equiv \frac{p(d, n)}{\left(\begin{array}{c}
2 n+d \\
d
\end{array}\right)}=\frac{1}{2^{d}}\left(\begin{array}{c}
2 d-1 \\
d
\end{array}\right)+\mathcal{O}\left(\frac{c_{d}}{n}\right)
$$

as the ratio of the upper bound $p(d, n)$ and the complexity $\left(\begin{array}{c}2 n+d \\ d\end{array}\right)$ of propagating multivariate Taylor series through a single multiplication operation. Hence, one obtains for large $d$

$$
r(d, n) \approx \frac{2^{d-1}}{\sqrt{\pi d}}+\mathcal{O}\left(\frac{c_{d}}{n}\right)
$$


TABLE 1. Nonvanishing coefficients for $d=2$

\begin{tabular}{|lll|r|}
\hline \multicolumn{3}{|c|}{ Multi-indices } & $c_{\mathbf{i}, \mathbf{j}}$ \\
\hline $\mathbf{i} \in M_{1}$ & $\mathbf{j} \in M_{2}$ & $\mathbf{i}^{T} \mathbf{j} \neq 0$ & $\frac{1}{2}$ \\
$\mathbf{i} \in M_{2}$ & $\mathbf{j} \in M_{2}$ & $\mathbf{i}^{T} \mathbf{j} \neq 0$ & $\frac{1}{2}$ \\
$\mathbf{i} \in M_{3}$ & $\mathbf{j} \in M_{2}$ & $\mathbf{i}^{T} \mathbf{j} \neq 0$ & $-\frac{1}{4}$ \\
$\mathbf{i} \in M_{3}$ & $\mathbf{j} \in M_{3}$ & $\mathbf{i}^{T} \mathbf{j}=2$ & 1 \\
\hline
\end{tabular}

As can be seen in Figure 3 the ratio $r(d, n)$ is quite small for the more important moderate values $d \leq 5$. It follows in particular that

$$
\begin{array}{ll}
r(1, n)=\frac{1}{2}-\frac{1}{4 n+2} & =\frac{1}{2}+\mathcal{O}\left(\frac{1}{n}\right), \\
r(2, n)=\frac{n(1+3 n)}{2(2 n+1)(n+1)} & =\frac{3}{4}+\mathcal{O}\left(\frac{1}{n}\right), \\
r(3, n)=\frac{n\left(1+3 n+5 n^{2}\right)}{(2 n+3)(2 n+1)(n+1)} & =\frac{5}{4}+\mathcal{O}\left(\frac{1}{n}\right),
\end{array}
$$

as well as

$$
\begin{aligned}
& r(4, n)=\frac{35}{16}+\mathcal{O}\left(\frac{1}{n}\right) \approx 2+\mathcal{O}\left(\frac{1}{n}\right), \\
& r(5, n)=\frac{63}{16}+\mathcal{O}\left(\frac{1}{n}\right) \approx 4+\mathcal{O}\left(\frac{1}{n}\right), \\
& r(6, n)=\frac{231}{32}+\mathcal{O}\left(\frac{1}{n}\right) \approx 7+\mathcal{O}\left(\frac{1}{n}\right), \\
& r(7, n)=\frac{429}{32}+\mathcal{O}\left(\frac{1}{n}\right) \approx 13+\mathcal{O}\left(\frac{1}{n}\right) .
\end{aligned}
$$

To apply the formula (19) at various points $\mathbf{x} \in \mathbb{R}^{\bar{n}}$ it makes sense to precompute the rational coefficients (18). So far a simple, explicit formula for them has not been found, but it is possible to further reduce the computation by grouping the additive terms together. A listing is given for $d=2$ and $d=3$.

When $d=2$ one has to consider the following four groups of multi-indices:

$$
\begin{aligned}
& M_{0}=\left\{\mathbf{0} \in \mathbb{N}_{0}^{n}\right\}, \\
& M_{1}=\left\{\mathbf{i} \in \mathbb{N}_{0}^{n}|| \mathbf{i} \mid=1\right\}, \\
& M_{2}=\left\{\mathbf{i} \in \mathbb{N}_{0}^{n}|| \mathbf{i} \mid=2 \text { and }|\operatorname{sign}(\mathbf{i})|=1\right\}, \\
& M_{3}=\left\{\mathbf{i} \in \mathbb{N}_{0}^{n}|| \mathbf{i} \mid=2 \text { and }|\operatorname{sign}(\mathbf{i})|=2\right\} .
\end{aligned}
$$

Then, the coefficient $c_{\mathbf{i}, \mathbf{j}}$ has to be calculated for each $\mathbf{i} \in M_{0} \cup M_{1} \cup M_{2} \cup M_{3}$ and each $\mathbf{j} \in M_{2} \cup M_{3}$. Table 1 lists the nonvanishing $c_{\mathbf{i}, \mathbf{j}}$. For $d=3$ there are three groups of multi-indices besides those of (22):

$$
\begin{aligned}
& M_{4}=\left\{\mathbf{i} \in \mathbb{N}_{0}^{n}|| \mathbf{i} \mid=3 \text { and }|\operatorname{sign}(\mathbf{i})|=1\right\}, \\
& M_{5}=\left\{\mathbf{i} \in \mathbb{N}_{0}^{n}|| \mathbf{i} \mid=3 \text { and }|\operatorname{sign}(\mathbf{i})|=2\right\}, \\
& M_{6}=\left\{\mathbf{i} \in \mathbb{N}_{0}^{n}|| \mathbf{i} \mid=3 \text { and }|\operatorname{sign}(\mathbf{i})|=3\right\} .
\end{aligned}
$$

Now, for each $\mathbf{i} \in M_{0} \cup \ldots \cup M_{6}$ and each $\mathbf{j} \in M_{4} \cup M_{5} \cup M_{6}$ the coefficients (18) must be considered. The nonvanishing $c_{\mathbf{i}, \mathbf{j}}$ are given by Table 2. As one can see, all coefficients $c_{\mathbf{i}, \mathbf{j}}$ for $|d| \leq 3$ are of moderate size and most are positive. It should also be noted that the interpolation procedure does not involve any divisions, so it does not expand errors occurred by propagating the univariate Taylor series. 
TABle 2. Nonvanishing coefficients for $d=3$

\begin{tabular}{|c|c|c|c|}
\hline Multi-indices & $c_{\mathbf{i}, \mathbf{j}}$ & Multi-indices & $c_{\mathbf{i}, \mathbf{j}}$ \\
\hline$\overline{\mathbf{i} \in M_{1} \quad \mathbf{j} \in M_{4} \quad \mathbf{i}^{T} \mathbf{j} \neq 0}$ & $\frac{1}{3}$ & $\overline{\mathbf{i} \in M_{5}} \quad \mathbf{j} \in M_{4} \quad \mathbf{i}^{T} \mathbf{j}=3$ & $\frac{2}{27}$ \\
\hline $\mathbf{i} \in M_{2} \quad \mathbf{j} \in M_{4}$ & $\frac{2}{9}$ & $\mathbf{i} \in M_{5} \quad \mathbf{j} \in M_{5} \quad \mathbf{i}^{T} \mathbf{j}=5$ & $\frac{2}{3}$ \\
\hline $\mathbf{j} \in M_{4} \quad \mathbf{i}^{T} \mathbf{j} \neq 0$ & $-\frac{5}{36}$ & $\mathbf{j} \in M_{5} \quad \mathbf{i}^{T} \mathbf{j}=4$ & $-\frac{1}{3}$ \\
\hline $\mathbf{i} \in M_{3} \quad \mathbf{j} \in M_{5}$ & $\frac{1}{4}$ & $\mathbf{i} \in M_{6} \quad \mathbf{j} \in M_{4} \quad \mathbf{i}^{T} \mathbf{j} \neq 0$ & $\frac{2}{27}$ \\
\hline $\mathbf{i} \in M_{4} \quad \mathbf{j} \in M_{4} \quad \mathbf{i}^{T} \mathbf{j} \neq 0$ & $\frac{2}{9}$ & $\mathbf{i} \in M_{6} \quad \mathbf{j} \in M_{5} \quad \mathbf{i}^{T} \mathbf{j}=3$ & $-\frac{1}{6}$ \\
\hline $\mathbf{i} \in M_{5} \quad \mathbf{j} \in M_{4}$ & $-\frac{5}{27}$ & $\mathbf{i} \in M_{6} \quad \mathbf{j} \in M_{6} \quad \mathbf{i}^{T} \mathbf{j}=3$ & 1 \\
\hline
\end{tabular}

\section{Some Run time Results}

This section presents some run time results for computing higher derivative tensors using the interpolation with univariate Taylor expansions described above.

To optimize bevel gears, exact knowledge of the geometric properties of bevel gear tooth flanks is necessary (see e.g. 9]). Arbitrary points on a given grid on the tooth flank can be calculated with machine accuracy by an analytical characterization [5] and computational differentiation. To that end one needs to compute the higher derivative tensors of a vector function $\mathbf{f}: \mathbb{R}^{3} \rightarrow \mathbb{R}^{3}$ describing a family of surfaces. A corresponding evaluation code consisting of approximately $160 \mathrm{C}$ statements was differentiated using ADOL-C [4] and a special driver for the calculation of higher derivative tensors by forward propagation of univariate Taylor series.

The run times observed on a Sun Sparc 10, always normalized by time for evaluating $\mathbf{f}$ without derivatives, are listed in Table 3. The third column states the theoretical run time ratios given by $(d+2)^{2}(d+1)^{2} / 4$ (see equation (44) in Section 3). The next column contains the ratios of the complete calculation of $\nabla^{k} \mathbf{f}(\mathbf{x})$, $k=0, \ldots, d$, using ADOL-C and the special driver mentioned above. As can be seen, the total ratio is about $50 \%-70 \%$ of the theoretical estimate. The ratio of the run time of the interpolation process only and the run time of the function evaluation is contained in the fourth column. In agreement with the asymptotic expansion for $r(d, n)$, one observes a doubling of the ratio with each increment of $d$ by 1 . Nevertheless, one finds on this example for $d \leq 9$ that no more than five percent of the run time for the derivative calculation is needed to interpolate the coefficients of the derivative tensors from the univariate Taylor series.

TABLE 3. Run-time ratios for derivative tensor evaluations

\begin{tabular}{|c|c|c|c|c|}
\hline$d$ & $\begin{array}{c}\text { distinct elements in } \\
\nabla^{k} \mathbf{f}(\mathbf{x}), k=0, \ldots, d\end{array}$ & $\begin{array}{c}\text { theoretical } \\
\text { run time ratio }\end{array}$ & $\begin{array}{c}\text { ratio for evaluating } \\
\nabla^{k} \mathbf{f}(\mathbf{x}), k=0, \ldots, d\end{array}$ & $\begin{array}{c}\text { ratio for the } \\
\text { interpolation }\end{array}$ \\
\hline 2 & 10 & 36 & 16.2 & 0.6 \\
3 & 20 & 100 & 45.0 & 1.9 \\
4 & 35 & 225 & 93.3 & 4.0 \\
5 & 56 & 441 & 184.7 & 8.5 \\
6 & 84 & 672 & 356.0 & 15.1 \\
7 & 120 & 1290 & 655.3 & 26.3 \\
8 & 165 & 2025 & 1174.0 & 44.4 \\
9 & 220 & 3025 & 2040.7 & 81.0 \\
\hline
\end{tabular}




\section{Conclusions}

Many applications in scientific computation require higher order derivatives. This article described a promising approach to compute higher order derivatives by using univariate Taylor series. The main result is equation (17), which represents general partial derivatives in terms of univariate Taylor coefficients.

It was found that the post-processing effort for the interpolation given by the function $p(d, n)$ in Section 4 is very small, especially for the more important moderate values of $d$. Also, for these $d$ the complexity ratio $q(d, n)$ analyzed in Section 3 between the new univariate Taylor approach and a more conventional multivariate Taylor approach is essentially 1 . In addition, the data structures and memory access pattern are much simpler and more regular, so that actual run-times should be significantly reduced.

\section{REFERENCES}

1. Christian H. Bischof, George F. Corliss, and Andreas Griewank, Structured second- and higher-order derivatives through univariate Taylor series. Optimization Methods and Software 2 (1993), 211-232.

2. Martin Berz, Differential algebraic description of beam dynamics to very high orders. Particle Accelerators 12 (1989), 109-124.

3. Martin Berz, Algorithms for higher derivatives in many variables with applications to bear physics. In George F. Corliss and Andreas Griewank, editors, Automatic Differentiation of Algorithms - Theory, Implementation, and Applications SIAM (1991), 147-156. MR 92h:65031

4. Andreas Griewank, David Juedes, and Jean Utke, ADOL-C: A package for the automatic differentiation of algorithms written in $C / C++$. ACM Trans. Math. Software 22 (1996), 131-167.

5. Andreas Griewank and George W. Reddien, Computation of cusp singularities for operator equations and their discretizations. J. Comput. Appl. Math. 26 (1989), 133-153. MR 91e:58036

6. Andreas Griewank, On automatic differentiation. In Masao Iri and K. Tanabe, editors, Mathematical programming: Recent developments and applications Kluwer Academic Publishers (1989), 83-108. MR 92k:65002

7. Andreas Griewank, Automatic evaluation of first- and higher-derivative vectors. In R. Seydel, F. W. Schneider, T. Küpper, and H. Troger, editors, Proceedings of the Conference "Bifurcation and Chaos: Analysis, Algorithms, Applications Birkhäuser (1991), 124-137. MR 91m:58001

8. Andreas Griewank, Computational differentiation and optimization. In J. Birge and K. Murty, editors, Mathematical Programming: State of the Art University of Michigan (1994), 102-131.

9. Ulf Hutschenreiter, A new method for bevel gear tooth flank computation. In Martin Berz, Christian Bischof, George F. Corliss and Andreas Griewank, editors, Computational Differentiation - Techniques, Applications, and Tools SIAM (1996), 161-172.

10. Rich Neidinger, An efficient method for the numerical evaluation of partial derivatives of arbitrary order. ACM Trans. Math. Software 18 (1992), 159-173. MR 93b:65040

Institute of Scientific Computing, Technical University Dresden, D-01062 Dresden, Germany

E-mail address: griewank@math.tu-dresden.de

Framework Technologies, Inc., 10 South Riverside Plaza, Suite 1800, Chicago, IlliNOIS 60606

E-mail address: utke@fti-consulting.com

Institute of Scientific Computing, Technical University Dresden, D-01062 Dresden, Germany

E-mail address: awalther@math.tu-dresden.de 